\title{
Correlation between the Diaphragmatic Contraction Pressure and the Slow Vital Capacity
}

\author{
Jae-Seok Lee $\cdot$ Dong-Wook Han - Tae-Wook Kang ${ }^{1 \dagger}$ \\ Department of Physical Therapy, Silla University \\ ${ }^{1}$ Department of Physical Therapy, Walk Rehabilitation Hospital
}

Received: May 29, 2019 / Revised: May 30, 2019 / Accepted: June 19, 2019

(C) 2019 J Korean Soc Phys Med

\section{| Abstract |}

PURPOSE: This study measured the external pressure on abdomen during maximal inspiration. The study determined the correlation between the diaphragmatic contraction pressure and the lung capacities to verify whether or not the measured pressure values can represent diaphragmatic contractility.

METHODS: The study included 32 healthy subjects (16 males and 16 females). The researchers fabricated their own diaphragmatic pressure belt (DiP Belt) to measure DCP. DiP Belt device was fixed on the front of the abdomen and the diaphragmatic contractility was measured during maximal inspiration. The lung capacities were measured using a portable digital spirometer device (Pony Fx, COSMED, Italy). A digital spirometer is a device that is used to test the flow of air entering and exiting the lungs.

RESULTS: DCP showed significant positive correlations with vital capacity (VC), inspiratory reserve volume (IRV)

†Corresponding Author : Tae-Wook Kang gornsapelr@naver.com, http://orcid.org/0000-0002-7755-404X

This is an Open Access article distributed under the terms of the Creative Commons Attribution Non-Commercial License (http://creativecommons.org/licenses/by-nc/3.0) which permits unrestricted non-commercial use, distribution, and reproduction in any medium, provided the original work is properly cited. and inspiratory capacity (IC). Among values of lung capacities, IC showed especially strong positive correlations with the DCP $(r=.714, p<.010)$. For the males, DCP showed significant positive correlations with IRV and IC, and DCP showed significant negative correlation with the expiratory reserve volume (ERV). For the females, DCP showed significant positive correlation with tidal volume (VT), but any significant correlation was not found with any of the other values of lung capacities.

CONCLUSION: DCP showed high correlations with IRV and IC associated with inspiratory capacity. Therefore, The DiP Belt can be looked upon as a simple device that is very useful for measuring diaphragmatic contractility.

Key Words: Diaphragm, Diaphragmatic Contraction Pressure, Vital Capacity

\section{Introduction}

The diaphragm muscle, which acts like a piston, is responsible for an essential role during respiration. When the dome-shaped diaphragm muscle moves downward, the thoracic cavity expands, pushing the internal organs below the diaphragm downward. Increased abdominal pressure moves the inner abdominal wall in an outward direction. As the airway opens, the lung volume then increases and 
the intrapleural pressure decreases, which enables effective breathing [1]. The diaphragm muscle is essential for inspiration, and this muscle can lose its muscular strength due to decreased contractility caused by aging, respiratory and neurological diseases, and prolonged mechanical ventilation [2-5].

There are currently various methods available in clinical practice for identifying the contractility of the diaphragm muscle. The non-invasive methods include measuring the oral or nasal inspiratory pressure. This method is primarily used in clinical practice for checking the maximal inspiratory muscle strength [6]. Other methods include measurement of the diaphragm mobility using X-ray and also measurement of the diaphragm mobility, thickness and thickening fraction by performing ultrasonography $[7,8]$. The invasive methods include inserting a pressure catheter down to the $1 / 3$ point of the esophagus to measure changes of the esophageal pressure during maximal sniffs (sniffPesmax) [6,9], as well as the method that measures the trans-diaphragmatic pressure (Pdi). For this method, a pressure catheter is inserted into the esophagus and stomach and the difference in the two pressures are then measured $[6,10]$.

However, the reliability of maximal inspiratory pressure measurements depends on the degree of cooperation from the patient, but it is very difficult to confirm which respiratory muscles cause such an effect. Pdi measurements require environmental conditions that most hospitals cannot provide, and skill and experience are required to use the catheter. Ultrasonography relies on how well the operator manipulates the equipment, but it can be difficult to obtain good-quality images from obese patients who display abdominal distension or those patients with extensive dressings [11].

Thus, there is the need for a simple method to identify diaphragmatic contractility. Accordingly, the present study measured the external pressure during diaphragmatic contraction, and this study determined the correlation between the diaphragmatic contraction pressure and the actual vital capacity (VC) to verify whether or not the measured pressure values can represent diaphragmatic contractility.

\section{Methods}

\section{Study population}

The sample size of the present study was calculated using the $\mathrm{G}^{*}$ power 3.1 program based on the results from a similar previous precedent study [7]. The calculated sample size was $\geq 31$ with a statistical power of $90.17 \%$. Based on the derived minimum sample size, the present study enrolled a total of 32 participants with 16 males and 16 females. Those people who are able to fully comply with the instructions provided and explained by the researchers were included in the study, while those who had experienced a respiratory infection during the past 2 weeks or those with limitations due to orthopedic surgery or pain were excluded.

\section{Measurement tools and methods}

\section{1) Diaphragmatic Pressure Belt (DiP Belt)}

To measure DCP, the researchers fabricated their own DiP Belt. The measurement principle was based on placing a hand on the lower abdomen during diaphragmatic respiration to feel for abdominal movement during inspiration by the subjects. Using this principle, a real-time monitoring pressure device was inserted inside a belt that was placed on the front of the abdomen, and the diaphragmatic contractility during maximum inspiration was checked. The fastening belt was used to affix the pressure measurement device on the body, and specifically on the upper abdomen area (the xiphoid process of the sternum). The fastening belt had a length of $124.5 \mathrm{~cm}$ and a height of $15 \mathrm{~cm}$, and a pressure cuff $(21.5 \mathrm{~cm} \times 12 \mathrm{~cm})$ was inserted into the inner-middle part of the belt. For 

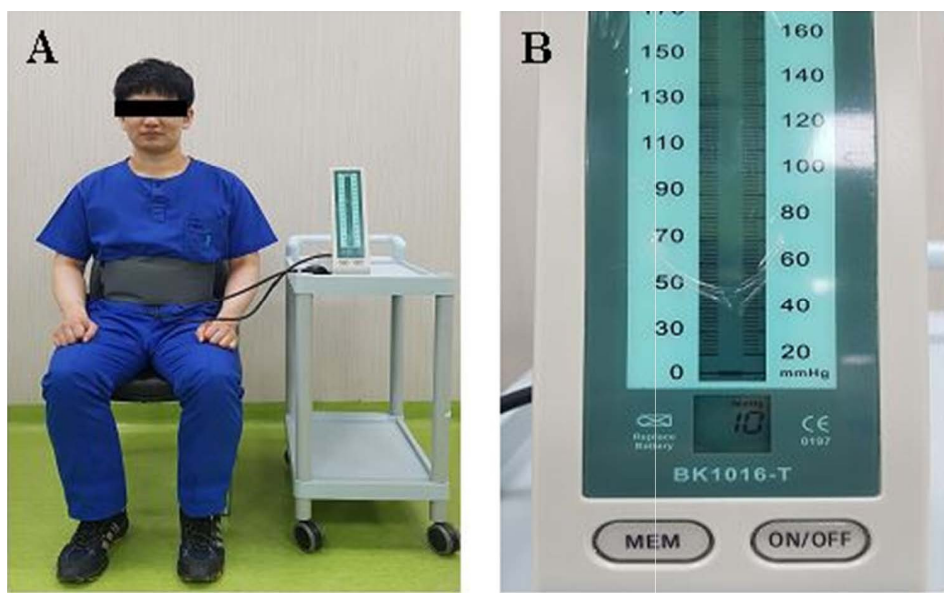

Fig. 1. Start position for measurement of the diaphragmatic contraction pressure using the DiP Belt. A: start position, B: reference pressure on resting $(10 \mathrm{mmHg})$
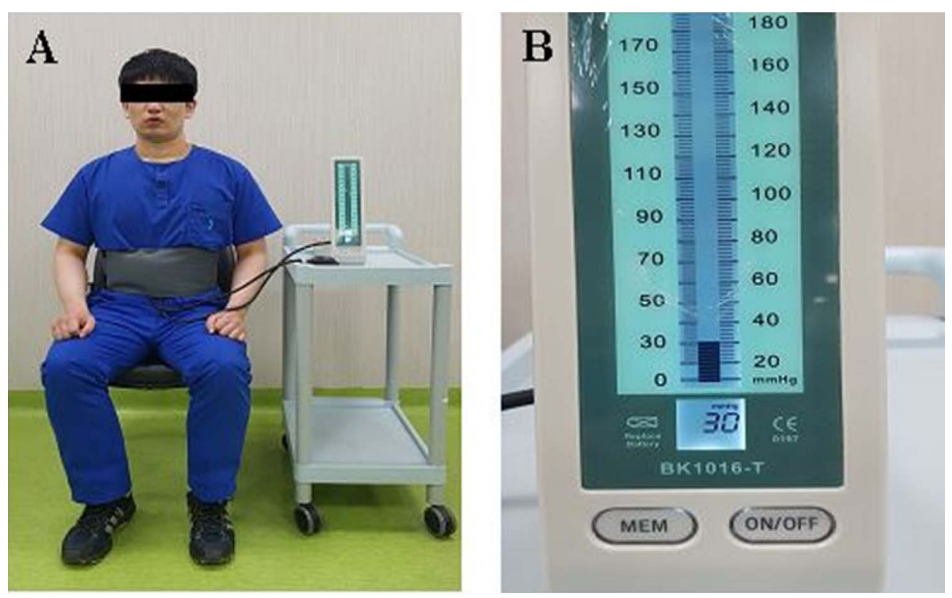

Fig. 2. Measurement of the maximal diaphragmatic contraction pressure using the DiP Belt. A: Pushing the DiP Belt with the maximum diaphragmatic breathing, B: Maximal diaphragmatic contraction pressure $(30 \mathrm{mmHg})$

obtaining real-time monitoring of changes of pressure, an electronic sphygmomanometer (BOKANG, China) was used to quantify the pressure changes. Because the thickness of the waist and abdomen vary from patient to patient, pressure during maximum expiration was set as the reference pressure $(10 \mathrm{mmHg})$ (Fig. 1). The researcher instructed the participants to "push and hold the pressure measurement device while breathing in as much air as possible" (Fig. 2). If elevated pressure values were found due to excessive lordosis of the lumbar region of the spine during maximum inspiration, then a measurement was taken again. The maximum and mean values from 3 repeated measurements were used in the analysis.

\section{2) Slow vital capacity (SVC)}

SVC was measured using a digital spirometer (Pony Fx, COSMED, Italy). A digital spirometer is a device that is used to test the flow of air entering and exiting the 
lungs. The measurement method involved having the participants bite on a mouthpiece while they were in a sitting position, followed by checking to make sure there are no air leaks, and then placing a nose clip on the nose. The researcher instructed the participants to relax the shoulders and breathe comfortably (tidal volume: VT), at least 3 times until their respiration became stable. After maximum expiration, the inspiratory vital capacity (IVC) was measured during maximum inspiration. The maximum VC value (VCmax) from 3 repeated measurements was used in the analysis. If the largest and the second largest values had a difference of $\geq .150 \mathrm{~L}$, then additional measurements were taken. Although 1 minute rest period is recommended for additional measurements, the present study used 2 minutes of relaxation before obtaining additional measurements [12].

\section{Statistical analysis}

Pearson correlation analysis was performed to examine the correlation between the value of the DiP Belt and the SVC. The SPSS WIN (ver. 22.0) statistics program was used in this study and the significance level was set to $a=.05$.

\section{Results}

\section{General characteristics of the participants}

The present study measured DCP and SVC of the 32 participants residing in $\mathrm{P}$ city. All the subjects were in their 20s, and 16 males and 16 females. The general characteristics of the participants were as follows (Table 1).

\section{Correlation between DCP and SVC}

DCP showed significant positive correlations with the following values among the SVC values: vital capacity (VC), inspiratory reserve volume (IRV), and inspiratory capacity (IC). Among these, the mean and maximum DCP showed strong positive correlations with IC $(r=.714, p<.01)$.
Table 1. General Characteristics of Subjects

(Unit)

\begin{tabular}{cc}
\hline Variable & Mean \pm SD \\
\hline Age & $25.156 \pm 3.673$ \\
Height $(\mathrm{cm})$ & $168.713 \pm 8.220$ \\
Weight $(\mathrm{kg})$ & $65.147 \pm 13.386$ \\
ADCP $(\mathrm{mmHg})$ & $39.614 \pm 11.599$ \\
PDCP $(\mathrm{mmHg})$ & $41.843 \pm 12.439$ \\
VC $(\ell)$ & $3.757 \pm .870$ \\
ERV $(\ell)$ & $1.275 \pm .384$ \\
VT $(\ell)$ & $.519 \pm .176$ \\
IRV $(\ell)$ & $1.962 \pm .737$ \\
IC $(\ell)$ & $2.462 \pm .765$ \\
\hline
\end{tabular}

ADCP: Average diaphragmatic contraction pressure, PDCP: Peak diaphragmatic contraction pressure, VC: Vital Capacity, ERV: Expiratory Reserve Volume, VT: Tidal Volume, IRV: Inspiratory Reserve Volume, IC: Inspiratory Capacity

Based on these findings, it was determined that a higher DCP meant a higher IC, which represents how much air was inhaled (Table 2).

\section{Correlation between DCP and SVC based on gender}

For the males, DCP showed significant positive correlations with IRV and IC, and significant negative correlation with the expiratory reserve volume (ERV). A higher DCP in males meant an increased IC due to greater diaphragmatic contractility, and as a result, the expiratory capacity of the lungs was also increased. For the females, DCP showed significant positive correlation with the VT, but significant correlation was not found with any of the other values (Table 3).

\section{Discussion}

DCP showed significant positive correlations with the total VC, IRV, and IC among the SVC values. These results 
Table 2. The Relationship Between Diaphragmatic Contraction Pressure and Slow Vital Capacity

\begin{tabular}{cccccc}
\hline Variable & VC $(\ell)$ & ERV $(\ell)$ & VT $(\ell)$ & IRV $(\ell)$ & IC $(\ell)$ \\
\hline ADCP $(\mathrm{mmHg})$ & $.589^{* *}$ & -.012 & .119 & $.673^{* *}$ & $.708^{* *}$ \\
PDCP $(\mathrm{mmHg})$ & $.597^{* *}$ & -.005 & .110 & $.680^{* *}$ & $.714^{* *}$ \\
\hline
\end{tabular}

${ }^{* *} \mathrm{p}<.010$. ADCP: Average diaphragmatic contraction pressure, PDCP: Peak diaphragmatic contraction pressure, VC: Vital Capacity, ERV: Expiratory Reserve Volume, VT: Tidal Volume, IRV: Inspiratory Reserve Volume, IC: Inspiratory Capacity

Table 3. The Relationship Between Diaphragmatic Contraction Pressure and Slow Vital Capacity According to Gender (Unit)

\begin{tabular}{ccccccc}
\hline Gender & Variable & VC $(\ell)$ & ERV $(\ell)$ & VT $(\ell)$ & IRV $(\ell)$ & IC $(\ell)$ \\
\hline \multirow{2}{*}{ Male } & ADCP $(\mathrm{mmHg})$ & .402 & $-.607^{*}$ & -.456 & $.732^{* *}$ & $.699^{* *}$ \\
& PDCP $(\mathrm{mmHg})$ & .426 & $-.583^{*}$ & -.472 & $.745^{* *}$ & $.709^{* *}$ \\
\hline \multirow{2}{*}{ Female } & ADCP (mmg) & .245 & .019 & $.525^{*}$ & .110 & .320 \\
& PDCP (mmH) & .229 & -.003 & $.512^{*}$ & .112 & .324 \\
\hline
\end{tabular}

${ }^{*} \mathrm{p}<.050,{ }^{* *} \mathrm{p}<.010$. ADCP: Average diaphragmatic contraction pressure, PDCP: Peak diaphragmatic contraction pressure, VC: Vital Capacity, ERV: Expiratory Reserve Volume, VT: Tidal Volume, IRV: Inspiratory Reserve Volume, IC: Inspiratory Capacity

indicate that a higher IC means a higher DCP; this represents the contractility of the diaphragm muscle, which is one of the main inspiratory muscles. Such results can be confirmed looking at how the values of SVC were defined. VT is defined as a comfortable respiratory flow, while inspiration including VT is defined as IC, and excluding VT is defined as IRV [12]. In the present study, IRV and IC showed the highest correlations with DCP, which can be viewed as demonstrating the contractility of the diaphragm muscle, a major inspiratory muscle. Among previous studies on VC based on the diaphragm's contractility, Rocha et al. [13] used X-ray to measure the mobility of the diaphragm and those researchers reported that diaphragm mobility in patients with chronic obstructive pulmonary disease (COPD) was positively correlated with $\mathrm{VC}$ and the strength of the inspiratory muscles, and among them, IC showed high positive correlation $(r=.80 ; p=.001)$ and showed negative correlation on the modified Medical Research Council (mMRC) dyspnea scale. Paulin et al. [14] reported that when diaphragm mobility was measured by ultrasonography, diaphragm mobility showed a positive correlation with distance of the 6-min walk test $(r=.38$; $\mathrm{p}=.005)$ and a negative correlation with the degree of dyspnea $(r=-.36 ; p=.007)$ in a 6 -minute walk test of the diaphragm, which indicated that diaphragm mobility affected the exercise capacity and dyspnea in patients with COPD. These previous studies reported that diaphragmatic contractility is correlated with VC, exercise capacity, and dyspnea. However, there are limitations when using X-ray or ultrasonography equipment to check the contractility of the diaphragm in clinical settings. Accordingly, for employing a simple and easy method to check the diaphragm's contractility, the present study used an external pressure device to check the DCP during maximum inspiration and this study demonstrated the correlation between DCP and IC.

Yet DCP of all the male participants showed positive correlations with values associated with IC, whereas DCP of the females showed correlation with only TV. Such findings are believed to be the result of differences in various respiration-related factors between males and females. A study by LoMauro and Aliverti [15] reported 
that females have a smaller airway diameter and lung volume, as well as a lower peak expiratory flow and a smaller diffusion surface than those of males, and that such differences in respiratory function between males and females was due to the anatomical differences based on the role of pregnancy that only females can have. Sawaya et al. [16] indicated that the skeletal muscle mass is correlated with $\mathrm{VC}$ and the strength of the respiratory muscles, and that the difference in $\mathrm{VC}$ and respiratory muscle strength between males and females was due to the difference of the skeletal muscle mass. Bellemare et al. [17] mentioned that females have structural differences as compared to those of males, such as a shorter diaphragm muscle and chest length. Kantarci et al. [18] reported that diaphragm mobility is affected by various factors, including gender, body weight, waist circumference, and age. When diaphragm mobility was measured by ultrasonography in a study by Boussuges et al. [19], the males showed significantly higher diaphragm mobility than did the females during both quiet breathing and deep breathing. Based on these previous studies, it can be believed that females have slight differences in anatomical structure and mass of their skeletal muscles, as compared to that of males. With respect to previous studies on the difference in breathing patterns between males and females, Ragnarsdottir and Kristinsdottir [20] reported that there was no difference of breathing patterns between males and females during quiet breathing, but that males showed greater abdominal breathing than females during deep breathing. Kaneko and Horie [21] used a three-dimensional motion analysis system to analyze the breathing pattern of males and females and they found that females showed greater thoracic cavity movement and less abdominal movement than males during quiet breathing. Based on these previous studies, it is thought that females have relatively lower diaphragm mobility than males when breathing, and to compensate for this, they tend to have a higher rate of chest breathing using other muscles besides the diaphragm muscle. Such breathing patterns of females caused the differences in the correlations between males and females to appear in the present study. Consequently, DCP measured by using the external pressure device showed a high correlation with the inspiratory flow and the DCP measured by using the external pressure device can be viewed as a useful method for checking the difference in diaphragmatic contractility.

However, since the study population in the present study consisted of healthy adults aged 20-29 years, it is difficult to generalize our findings for people of all ages and patients suffering with other diseases. Moreover, since the validity of the diaphragm contractility was confirmed through a digital spirometer, the study has the limitation of not confirming diaphragm mobility by using actual images. Therefore, additional future studies are needed with the study population consisting of diverse age groups and patients with various respiratory diseases, while diaphragm contractility should also be compared to the actual values measured by ultrasonography and X-ray.

\section{Conclusion}

The DiP Belt was used as a simple method in the study to visualize diaphragmatic contractility DCP showed high correlations with IRV and IC associated with inspiratory flow. Therefore, The DiP Belt can be looked upon as a simple device that is very useful for checking diaphragmatic contractility.

\section{References}

[1] De Troyer A, Boriek AM. Mechanics of the respiratory muscles. Compr Physiol. 2011;1(3):1273-300.

[2] Simões RP, Castello V, Auad MA, et al. Prevalence of reduced respiratory muscle strength in institutionalized elderly people. Sao Paulo Med J. 2009;127(2):78-83.

[3] Laghi F, Tobin MJ. Disorders of the respiratory muscles. Am J Respir Crit Care Med. 2003;168(1):10-48. 
[4] Kim M, Lee K, Cho J, et al. Diaphragm Thickness and Inspiratory Muscle Functions in Chronic Stroke Patients. Med Sci Monit. 2017;11(23):1247-53.

[5] Dres M, Goligher EC, Heunks LMA, et al. Critical illness-associated diaphragm weakness. Intensive Care Med. 2017;43(10):1441-52.

[6] ATS/ERS (American Thoracic Society/European Respiratory Society) Statement on respiratory muscle testing. Am J Respir Crit Care Med. 2002;166(4):518-624.

[7] Cardenas LZ, Santana PV, Caruso P, et al. Diaphragmatic Ultrasound Correlates with Inspiratory Muscle Strength and Pulmonary Function in Healthy Subjects. Ultrasound Med Biol. 2018;44(4):786-93.

[8] Santana PV, Prina E, Albuquerque AL, et al. Identifying decreased diaphragmatic mobility and diaphragm thickening in interstitial lung disease: the utility of ultrasound imaging. J Bras Pneumol. 2016;42(2):88-94.

[9] Laroche CM, Carroll N, Moxham J, et al. Clinical significance of severe isolated diaphragm weakness. Am Rev Respir Dis. 1988;138(4):862-6.

[10] Steier J, Kaul S, Seymour J, et al. The value of multiple tests of respiratory muscle strength. Thorax. 2007; 62(11):975-80.

[11] Caruso P, Albuquerque AL, Santana PV, et al. Diagnostic methods to assess inspiratory and expiratory muscle strength. J Bras Pneumol. 2015;41(2):110-23.

[12] Miller MR, Hankinson J, Brusasco V, et al. Standardisation of spirometry. Eur Respir J. 2005;26(2):319-38.

[13] Rocha FR, Brüggemann AK, Francisco DS, et al. Diaphragmatic mobility: relationship with lung function, respiratory muscle strength, dyspnea, and physical activity in daily life in patients with COPD. J Bras Pneumol. 2017;43(1):32-7.

[14] Paulin E, Yamaguti WP, Chammas MC, et al. Influence of diaphragmatic mobility on exercise tolerance and dyspnea in patients with COPD. Respir Med. 2007; 101(10):2113-8.

[15] LoMauro A, Aliverti A. Sex differences in respiratory function. Breathe. 2018;14(2):131-140.

[16] Sawaya Y, Ishizaka M, Kubo A, et al. Correlation between skeletal muscle mass index and parameters of respiratory function and muscle strength in young healthy adults according to gender. J Phys Ther Sci. 2018;30(12):14247.

[17] Bellemare F, Jeanneret A, Couture J. Sex differences in thoracic dimensions and configuration. Am J Respir Crit Care Med. 2003;168(3):305-12.

[18] Kantarci F, Mihmanli I, Demirel MK, et al. Normal diaphragmatic motion and the effects of body composition: determination with M-mode sonography. J Ultrasound Med. 2004;23(2):255-60.

[19] Boussuges A, Gole Y, Blanc P. Diaphragmatic motion studied by m-mode ultrasonography: methods, reproducibility, and normal values. Chest. 2009;135(2):391-400.

[20] Ragnarsdóttir M, Kristinsdóttir EK. Breathing movements and breathing patterns among healthy men and women 20-69 years of age. Reference values. Respiration. 2006;73(1):48-54.

[21] Kaneko H, Horie J. Breathing movements of the chest and abdominal wall in healthy subjects. Respir Care. 2012;57(9):1442-51. 